\title{
CONSUMO DE TABACO, ÁLCOOL E DROGAS EM JOVENS ESTUDANTES
}

\author{
Eugénia Maria Garcia Jorge Anes \\ Escola Superior de saúde de Bragança, IPB \\ eugenia@ipb.pt \\ Celeste da Cruz Meirinho Antão \\ Escola Superior de saúde de Bragança, IPB \\ celeste@ipb.pt
}

Fecha de Recepción: 23 Febrero 2018

Fecha de Admisión: 10 Abril 2018

\section{RESUMO}

Os comportamentos da adolescência expõem muitas vezes o jovem a uma sucessão de riscos, em especial, ao consumo de substâncias, com consequentes implicações para a saúde e normal desenvolvimento dos jovens. Pretendemos analisar o comportamento dos jovens estudantes em relação ao consumo de substâncias. É um estudo descritivo, transversal e analítico com uma abordagem quantitativa. 0 instrumento de colheita de dados é constituído pela Global School-Based Studant Health Survey (GSHS), ao qual foram associadas questões de caraterização. A amostra é constituída por 212 jovens estudantes. Maioritariamente feminina (54,7\%), com idades compreendidas entre os 12 e os 18 anos, e vive com ambos os pais. (72,2\%). Frequentam entre $07^{\circ}$ e $012^{\circ}$ ano de escolaridade. Referem ter iniciado consumo de álcool $62,7 \%$ dos estudantes; com inicio antes dos 13 anos (28,8\%); no último mês, 32,5\% dos adolescentes consumiram-no pelo menos um ou dois dias; beberam mais de 5 bebidas, $11,3 \%$; compram-no em bares, no mercado ou na rua; já se embebedaram $22,6 \%$. e; em $58,5 \%$ das famílias existe pelo menos um consumidor de álcool. Já fumaram $31,1 \%$ dos inquiridos; iniciaram depois dos 16 anos (10,8\%); dizem ter fumado nos últimos 30 dias $8,5 \%$. 0 consumo de drogas é mais baixo (4,7\%) quando comparado com 0 álcool e o tabaco. Iniciam maioritariamente o consumo entre os 12 e os 13 anos; no último mês foi consumida por 4,3\%; é comprada ou adquirida através de amigos ou familiares. Foi verificada associação entre os consumos e a existência de amigos e pais consumidores e; entre os consumos e 0 já ter tido relações sexuais. É realçada neste trabalho a importância da família e dos amigos, como determinantes de consumos. 0 conhecimento destes determinantes é de fulcral importância na programação e implementação de medidas preventivas, pelo que devem ser tidas em conta ao nível da tomada de decisão.

Palavras-chave: jovem; consumo; álcool; tabaco; drogas 


\section{CONSUMO DE TABACO, ÁLCOOL E DROGAS EM JOVENS ESTUDANTES}

\section{ABSTRACT}

\section{Consumption of tobacco, alcohol and drugs in young students.}

The behavior of adolescence often leads young people to risks, especially substance use, with implications for the health and normal development of young people. We intend to analyze the behavior of young students in relation to substance use. It is a descriptive, transversal and analytical study, with a quantitative approach. The data collection instrument shall consist of the Global School-Based Studying Health Survey (GSHS), to which characterization issues were associated. The sample is made up of 212 young students. Mostly female (54.7\%), between the ages of 12 and 18 , and lives with both parents. $(72.2 \%)$. They attend between the 7 th and 12 th grades. They reported having started drinking alcohol $62.7 \%$ of the students; with onset before the age of $13(28.8 \%)$; in the last month, $32.5 \%$ of adolescents consumed it for at least one or two days; drank more than 5 drinks, $11.3 \%$; they buy it in bars, in the market or in the street; already got drunk $22.6 \%$. and; in $58.5 \%$ of households there is at least one alcohol consumer. Already smoked $31.1 \%$ of respondents; started after 16 years (10.8\%); said to have smoked in the last 30 days $8.5 \%$. Drug use is lower $(4.7 \%)$, when compared to alcohol and tobacco consumption. Most of them start consumption between the ages of 12 and 13; in the last month was consumed by $4.3 \%$; is purchased or acquired through friends or family. Association between consumptions and the existence of friends and consumer parents was verified. The importance of family and friends as determinants of consumption is highlighted in this paper. Knowledge of these determinants is of paramount importance in the planning and implementation of preventive measures and should therefore be taken into account at the decision-making level.

Keywords: young; consumption; alcohol; tobacco; drugs

\section{INTRODUÇÃO}

As atitudes e os comportamentos incluídos no estilo de vida dos adolescentes podem influenciar, directa ou indirectamente, a sua saúde a curto ou a longo prazo. A maioria das actuais ameaças à saúde dos adolescentes são a consequência de factores sociais, ambientais e comportamentais (DiClemente, Hansen \& Ponton, 1996). Podem designar-se estes como factores de risco e incluem um largo espectro de comportamentos e efeitos relacionados como seja o consumo de substâncias, violência, suicídio, gravidez na adolescência, álcool, distúrbios de alimentação, e doenças sexualmente transmissíveis (DiClemente, Hansen \& Ponton, 1996; Lerner \& Thompson 2002).

É necessário promover a autonomia, a responsabilização e a participação activa dos jovens na construção da sua vida, ajudando-os a enfrentá-la sem recurso a comportamentos desviantes, como seja o consumo de substâncias aditivas, a violência e a sexualidade de risco (Matos et al., 2003; Matos, 2005).

A adolescência é caraterizado como um período de profundas mudanças, das quais surge uma série de comportamentos que muitas vezes expõem o jovem a uma sucessão de riscos, nomeadamente, ao consumo de substâncias (Matos, 2008).

Geralmente, nos primeiros consumos encontra-se o tabaco, constituiindo a primeira droga consumida pelas crianças e adolescentes (American Academy of Pediatrics, 2001). É unanime entre os autores consultados, que o comportamento tabágico se inicia na maior parte das vezes na adolescência e poucas pessoas se tornam fumadoras depois dos 18 anos. Este consumo aumenta potencialmente nos adolescentes que têm outros comportamentos de risco, como o consumo de drogas ilícitas e álcool (American Academy of Pediatrics, 2001). 0 consumo de tabaco por parte dos pais, irmãos e amigos, é também considerado fator potenciador do consumo de tabaco.

Mundialmente, 0 álcool constitui a substância psicoativa mais consumida e simultaneamente a droga de eleição entre crianças e adolescentes (WHO, 2001; WHO, 2004). 
Além da alta prevalência do consumo de álcool por adolescentes, dois outros fatores são relevantes: a idade de início do uso de álcool e o padrão de consumo (Vieira, Ribeiro, Romano, \& Laranjeira, 2007).

Alguns estudos têm demostrado que a idade de início do consumo é cada vez mais precoce (Galduroz, Noto, Nappo \& Carlini, 2004).

Por outro lado, à medida que aumenta a precocidade da experimentação do álcool, mais graves se tornam as consequências e maior o risco de desenvolvimento de abuso e dependência do mesmo (Meloni \& Laranjeira, 2004).

Esta população é sensível aos resultados negativos, e muitas vezes trágicos, do consumo de álcool (Reboussin, Song, Shrestha, Lohman \& Wolfson, 2006).

Nos Estados Unidos, são atribuídas ao álcool as primeiras quatro causas de morte entre jovens na faixa etária entre os 10 e os 24 anos, como sejam os acidentes de viação, lesões não intencionais, homicídios e suicídios (Galduroz, Noto, Fonseca \& Carlini, 2004).

Em termos de padrão de consumo, ele é caraterístico nesta população. Quando bebem os adolescentes, tendência é fazê-lo de forma pesada, exibindo episódios agudos de abuso, também conhecido por "binge drinking", ou seja, beber cinco ou mais doses em uma ocasião (Hingson \& Winter, 2003). Este comportamento aumenta o risco de uma sucessão de problemas sociais e de saúde, como doenças sexualmente transmissíveis, gravidez indesejada, enfarte do miocárdio, acidentes de viação, alterações de comportamento, violência e lesões não intencionais (Reboussin, Song, Shrestha, Lohman \& Wolfson, 2006).

A perceção do risco para a saúde relativamente ao consumo regular de álcool a maior parte dos jovens portugueses entre os $15-24$ anos foi considerada de alto risco (59\%), para o consumo ocasional a maior parte considerou-o de baixo risco (68\%) (DGS, 2016). Nos dois tipos de consumo os valores Portugueses são semelhantes aos da média europeia (DGS, 2016).

Relativamente ao consumo de outras substâncias nocivas à saúde, este pode ser influenciado por diversos fatores como a idade, a etnia, a estrutura familiar, o estatuto socioeconómico da família, atitudes de consumo da família e dos amigos, ligação com a família e com os amigos, fraco envolvimento escolar ou profissional, sintomas de stress, ansiedade e depressão, problemas de autoestima e outras características da personalidade (Matos,2008b).

Os grupos e os amigos constituem um enorme reforço ou determinante no que respeita ao consumo de substâncias. 0 seu consumo pode ser reforçado socialmente pela sua presença e em especial dos chamados amigos íntimos (Prinstein, Boergers \& Spirito, 2001).

Tavares, Béria e Lima (2004) afirmam que os adolescentes que consideravam ter um pai liberal consomem mais substâncias, em comparação com os que consideravam o pai moderado ou autoritário. A forma como a liberdade é concedida assume especial relevância para a conquista da maturidade. Muitas vezes, incompreensão e rejeição encontram-se mascaradas debaixo da concessão de uma excessiva liberdade que 0 adolescente vive. Outros factos considerados preditores de consumo pelos mesmos autores é a situação conjugal dos pais, onde os filhos de pais separados, apresentam significativamente maior consumo de drogas em relação com aqueles cujos pais viviam juntos; Uso de drogas e uso de álcool por parte dos pais; os maus tratos na adolescência também estão relacionados elevado o uso de drogas e consequentemente a violência (Tavares, Béria \& Lima, 2004).

Os adolescentes que referem consumir substâncias apresentam mais comportamentos ligados ao risco e um relacionamento menos positivo com os seus contextos (Matos, 2008b).

Existem diferenças de género quando estão em causa consumos, os rapazes tendem a consumir no contexto de atividades e as raparigas mais num contexto de partilha de intimidade, sendo 


\section{CONSUMO DE TABACO, ÁLCOOL E DROGAS EM JOVENS ESTUDANTES}

que em ambos os géneros o consumo de álcool e drogas está associado à procura de intimidade nas relações interpessoais (Malow-Iroff, 2006).

\section{OBJETIVO}

0 presente estudo pretende estudar e analisar os comportamentos dos jovens estudantes do terceiro ciclo e secundário de uma escola da cidade de Bragança (Portugal) em relação ao consumo de substâncias, nomeadamente tabaco, álcool e drogas.

\section{METODOLOGIA}

É um estudo descritivo, transversal e analítico com uma abordagem quantitativa. A população do presente estudo é constituída por todos os alunos do terceiro ciclo e secundário de uma escola Secundária do Nordeste de Portugal.

A seleção da amostra foi probabilística por Conglomerado "cluster sampling", no universo dos jovens do terceiro ciclo e secundário da Escola. A amostra por conglomerados é uma técnica probabilística na qual as unidades amostrais são grupos (clusters) de elementos. Mais do que representativos de subconjuntos populacionais, os conglomerados devem ser carateristicos da população total. 0 "cluster" ou unidade de análise é a turma. De modo a obter uma amostra representativa da população escolar, o número de unidades de análise será equitativo para cada ano de escolaridade.

Do total de 559 estudantes, foram selecionadas aleatoriamente dois "clusters" (turmas) por ano, de onde resultaram 290 estudantes. Foi solicitada consentimento informado por escrito à instituição e em particular a cada estudante e encarregado de educação. Resultando uma amostra de 212 estudantes, correspondendo a $37,9 \%$ da população.

Os estudantes foram informados do carater voluntário da sua participação, podendo recusá-la em qualquer momento, sem que tal fato traga consequências. Foram também esclarecidos acerca do anonimato e confidencialidade dos dados, sendo a informação recolhida utilizada exclusivamente para 0 estudo da temática, de forma a contribuir para uma base de intervenção ao nível da promoção da saúde e investigação.

0 questionário foi auto-preenchido por cada estudante, sem a intervenção do investigador. A recolha de dados decorreu no primeiro trimestre de 2012.

0 instrumento de colheita de dados é constituído pela Global School-Based Studant Health Survey (GSHS), ao qual foram associadas questões de caraterização (sexo, idade, ciclo de escolaridade, com quem habita, atividade física e $\mathrm{n}^{\circ}$ de horas de sono diárias).

A Escala Mundial de Saúde Escolar (Global School-based Student Health survey - GSHS) foi desenvolvida pela Organização Mundial da Saúde (OMS) em colaboração com as Nações Unidas (United Nations Children's Fund-UNICEF, United Nations Educational, Scientific and Cultural Organization-UNESCO e Joint United Nations Program on HIV/AIDS-UNAIDS) e com a assistência técnica do CDC (Centers for disease control and Prevention). Foi solicitada autorização ao Centers for disease control and Preventio. São aqui analisados os módulos relativos ao consumo de substancias

Para o tratamento de dados utilizou-se como recurso o programa informático SPSS-v23 (Statistical Package for the Social Science - v23). Foi utilizada estatística descritiva e inferencial. Todos os dados foram tratados em termos globais em relação às variáveis da escala e variáveis de caraterização.

\section{RESULTADOS}

A mostra do presente estudo é constituída por 212 jovens adolescentes e é maioritariamente 
feminina $(54,7 \%)$. Apresentam idades compreendidas entre os 12 e os 18 anos, com uma média de idade de 14,91 anos (desvio padrão de 1,70 anos). Relativamente ao ano de escolaridade, o mais representativo é o $10^{\circ}$ ano, o menos representativo é $07^{\circ}$ ano. Por questões de operacionalidade, esta variável foi recodificada em $3^{\circ}$ ciclo e secundário. A grande maioria dos adolescentes vive com ambos os pais $(72,2 \%)$, só com a mãe vivem $11,8 \%$ e com amigos vivem 11,3 . Constatamos que mais de metade da nossa amostra $(57,5 \%)$ pratica exercício físico fora da escola. 0 número de horas de sono por noite, onde podemos constatar que uma elevada percentagem $(45,3 \%)$ dos estudantes dorme menos de 8 horas de sono por noite.

\section{Uso de substâncias}

Relativamente ao consumo de tabaco, 68,9\% dos estudantes afirmam nunca ter fumado. No grupo dos que já fumaram, a idade do primeiro consumo referenciada mais frequentemente é a partir dos 16 anos $(10,8)$. Quando analisado o consumo de tabaco nos últimos 30 dias, constatamos que a percentagem de adolescentes que não fumou aumentou para 91,5\%. 0 número de dias de consumo de tabaco é variável, o mais frequente é um ou dois dias e 6 a 9 dias com uma percentagem de $2,8 \%$ em ambos os casos. Com menor percentagem (1,4\%) temos estudantes que fumam diariamente. Quando questionados acerca de uma possível tentativa de deixar de fumar nos últimos 12 meses, 89,1\% afirmam não ter fumado no último ano. Tentaram deixar de fumar 7,1\% dos participantes. No que respeita ao consumo de tabaco por parte de outras pessoas na presença dos inquiridos nos últimos 7 dias, 59,9\% dos jovens afirmam que sim, que outras pessoas fumaram na sua presença. 0 número de dias em que se verificaram estes consumos é variável, com maior representatividade ocorreu um dia $(24,5 \%)$, seguido de 7 dias $(15,6 \%)$. Mais de metade dos pais ou encarregados de educação não fuma (68,9\%). 0 pai surge como 0 elemento mais fumador (16,5\%).

No que respeita 0 consumo de álcool, 62,7\% dos estudantes já 0 consumiram. A idade em iniciaram é abaixo dos 13 anos para 28,8\%. Entre os 14 e os 15 anos iniciaram 0 consumo 22,2\%. Já acima dos 16 anos apenas referem tê-lo iniciado 11,8\%. Relativamente ao consumo de álcool no último mês $32,5 \%$ dos adolescentes consumiram-no pelo menos um ou dois dias. É importante referir no entanto que, cerca de 10\% consumiram-no entre 3 a 9 dias. Em termos de quantidade $11,3 \%$ beberam mais de 5 bebidas. 0 acesso ao álcool apresenta formas diversas, sendo de destacar que aproximadamente $27 \%$ das crianças dizem comprá-Io, com menor representatividade segue a sua aquisição através de amigos e no próprio domicílio com 2,4\%, respetivamente. Ser o próprio jovem a produzi-lo é um acesso pouco frequente $(1,4 \%)$. Dos inquiridos, $22,6 \%$ afirmam já se terem embebedado. Quando solicitados a contabilizar as bebedeiras, metade destes fizeram-nos uma ou duas vezes $(11,3 \%)$ e da outra metade, $4,2 \%$ fizeram-no mais de 10 vezes. $2,9 \%$ dos consumidores de álcool dizem já ter tido problemas relacionados com o consumo, com a família ou amigos, faltaram à escola e estiveram envolvidos em brigas. Relativamente ao consumo de álcool por parte da família, constatámos que em $58,5 \%$ das famílias existe pelo menos um consumidor, com maior percentagem para o pai com $37,3 \%$, seguido de ambos os progenitores ou tutores com $17 \%$.

Relativamente ao consumo de drogas, a quase totalidade dos alunos nunca consumiu drogas $(95,3 \%)$ durante a sua vida. Fizeram-no uma ou duas vezes $4,2 \%$. Nos inquiridos que já consumiram drogas $(4,7 \%)$, fizeram-no pela primeira vez entre os 12 e 13 anos $(3,3 \%)$ e depois dos 16 anos $(1,4 \%)$. Durante 0 último ano consumiram drogas $4,1 \%$ dos estudantes, usaram drogas uma ou duas vezes $2,4 \%$ e mais de 10 vezes 1,9\%. Acessaram à droga através de amigos 3,3\% e obtiveram-na na família $0,9 \%$.

Foi verificada associação entre o consumo de tabaco e a presença de pessoas fumadoras. Na tabela 1 apresentamos as frequências observadas e as esperadas, a estatística do teste e 0 valor de 


\section{CONSUMO DE TABACO, ÁLCOOL E DROGAS EM JOVENS ESTUDANTES}

prova no teste de associação do qui-quadrado entre o consumo de tabaco e outras pessoas fumarem na presença do estudante. 0 valor de prova obtido é inferiora $5 \%$ pelo que as variáveis estão estatisticamente associadas. Da análise das frequências observadas e esperadas, um maior número de estudantes fuma quando outras pessoas fumam na sua presença, e vice-versa, se outras pessoas não fumam na sua presença um maior número de estudantes também não fuma.

\section{Tabela 1}

Teste de associação do qui-quadrado entre o consumo de tabaco $e$ outras pessoas terem fumado na presença do estudante

\begin{tabular}{|c|c|c|c|c|c|c|}
\hline \multirow{2}{*}{\multicolumn{3}{|c|}{$\begin{array}{l}\text { Associação entre o consumo de } \\
\text { tabaco e a presença de outos } \\
\text { fumadores junto do estudante }\end{array}$}} & \multicolumn{2}{|c|}{$\begin{array}{c}\text { Outras pessoas } \\
\text { fumaram na tua } \\
\text { presença }\end{array}$} & \multirow[t]{2}{*}{ ET } & \multirow{2}{*}{$\begin{array}{l}\text { Valor } \\
\text { de } \\
\text { prova }\end{array}$} \\
\hline & & & Não & Sim & & \\
\hline \multirow{4}{*}{ 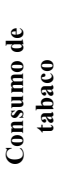 } & Nunca & Observado & 76 & 70 & \multirow{4}{*}{26,355} & \multirow{4}{*}{$<0,001$} \\
\hline & fumei & Esperado & 58,5 & 87,5 & & \\
\hline & \multirow{2}{*}{$\begin{array}{c}\text { Já } \\
\text { fumei }\end{array}$} & Observado & 9 & 57 & & \\
\hline & & Esperado & 26,5 & 39,5 & & \\
\hline
\end{tabular}

Na tabela 2 apresentamos as frequências observadas e as esperadas, a estatística do teste e 0 valor de prova no teste de associação do qui-quadrado entre o consumo de tabaco e pais ou encarregado de educação fumarem. 0 valor de prova obtido é inferiora $5 \%$ pelo que as variáveis estão estatisticamente associadas. Da análise das frequências observadas e esperadas, um maior número de estudantes fuma quando os pais fumam, e vice-versa, se os pais não fumam um maior número de estudantes também não fuma face ao que era esperado.

Tabela 2

Testes de associação do qui-quadrado entre o consumo de tabaco e pais ou encarregados de educação fumadores

\begin{tabular}{|c|c|c|c|c|c|c|}
\hline \multirow{2}{*}{\multicolumn{3}{|c|}{$\begin{array}{l}\text { Associação entre o consumo de } \\
\text { tabaco e a existência de pais ou } \\
\text { encarregados de educação } \\
\text { fumadores }\end{array}$}} & \multicolumn{2}{|c|}{ Os pais fumam } & \multirow{2}{*}{ ET } & \multirow{2}{*}{$\begin{array}{c}\text { Valor } \\
\text { de } \\
\text { prova }\end{array}$} \\
\hline & & & Não & Sim & & \\
\hline \multirow{4}{*}{ 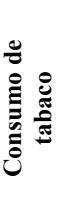 } & Nunca & Observado & 113 & 33 & \multirow{4}{*}{14,661} & \multirow{4}{*}{$<0,001$} \\
\hline & fumei & Esperado & 100,5 & 45,5 & & \\
\hline & \multirow{2}{*}{$\begin{array}{c}\text { Já } \\
\text { fumei }\end{array}$} & Observado & 33 & 33 & & \\
\hline & & Esperado & 45,5 & 20,5 & & \\
\hline
\end{tabular}

Na tabela 3 apresentamos as frequências observadas e as esperadas, a estatística do teste e 0 valor de prova no teste de associação do qui-quadrado entre o ter relações sexuais e o consumo de álcool. 0 valor de prova obtido é inferiora $5 \%$ pelo que as variáveis estão estatisticamente associadas. Da análise das frequências observadas e esperadas, conclui-se que o número de estudantes 
que nunca bebeu e que nunca teve relações sexuais é superior ao esperado. E que, estudantes que já beberam e já tiveram relações sexuais são em número superior ao esperado. Era também esperado um maior número de estudantes que já beberam e não tiveram relações sexuais.

Tabela 3

Teste de associação do qui-quadrado entre o ter relações sexuais e o consumo de álcool

\begin{tabular}{|c|c|c|c|c|c|c|}
\hline \multirow{2}{*}{\multicolumn{3}{|c|}{$\begin{array}{c}\text { Associação entre os pais ouvirem as } \\
\text { preocupações dos filhos e o sentir-se } \\
\text { só }\end{array}$}} & \multicolumn{2}{|c|}{ Consumo de álcool } & \multirow[b]{2}{*}{ ET } & \multirow{2}{*}{$\begin{array}{c}\text { Valor } \\
\text { de } \\
\text { prova }\end{array}$} \\
\hline & & & $\begin{array}{c}\text { Nunca } \\
\text { bebi }\end{array}$ & Já bebi & & \\
\hline \multirow{4}{*}{ 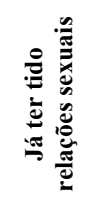 } & & Observado & 9 & 56 & \multirow{4}{*}{20,569} & \multirow{4}{*}{$<0,001$} \\
\hline & & Esperado & 24,2 & 40,8 & & \\
\hline & \multirow{2}{*}{ Não } & Observado & 70 & 77 & & \\
\hline & & Esperado & 54,8 & 92,2 & & \\
\hline
\end{tabular}

A relação entre 0 ter relações sexuais e o consumo de tabaco é também estatisticamente significativa pois 0 valor de prova obtido é inferior a $5 \%$. 0 número de estudantes que já fumaram e tiveram relações sexuais é superior ao esperado, mas também se observou um maior número de estudantes que nunca fumaram nem tiveram relações sexuais superiores ao esperado (Tabela 4).

Tabela 4

Teste de associação do qui-quadrado entre o ter relações sexuais e o consumo de tabaco

\begin{tabular}{|c|c|c|c|c|c|c|}
\hline \multirow{2}{*}{\multicolumn{3}{|c|}{$\begin{array}{l}\text { Associação entre os pais ouvirem as } \\
\text { preocupações dos filhos e o sentir-se } \\
\text { só }\end{array}$}} & \multicolumn{2}{|c|}{ Consumo de tabaco } & \multirow{2}{*}{ ET } & \multirow{2}{*}{$\begin{array}{c}\text { Valor de } \\
\text { prova }\end{array}$} \\
\hline & & & Nunca & Já & & \\
\hline \multirow{4}{*}{ 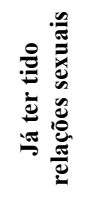 } & Sim & Observado & 33 & 32 & \multirow{4}{*}{13,130} & \multirow{4}{*}{$<0,001$} \\
\hline & & Esperado & 44,8 & 20,2 & & \\
\hline & \multirow{2}{*}{ Não } & Observado & 113 & 34 & & \\
\hline & & Esperado & 101,2 & 45,8 & & \\
\hline
\end{tabular}

\section{DISCUSSÃO}

Este estudo pretendeu estudar os comportamentos dos estudantes relativamente ao consumo de tabaco álcool e drogas, analisando a associação entre as variáveis sociodemográficas.

Resultou uma amostra com perfil semelhante a outros efetuados a nível nacional, maioritariamente feminina, com idades compreendidas entre os 12-18 anos e a viver com ambos os pais (Matos, Simões, Camacho, \& Reis, 2014). A prática de atividade física fora da escola é efetuada por mais de metade da amostra, evidenciando uma prática superior ao encontrado no estudo nacional da Aventura Social em 2014 (Matos, Simões, Camacho, \& Reis, 2014). 0 número de horas de sono é reduzido e inferior a 8 para quase metade dos estudantes resultados piores a estudos prévios (Hershner \& Chervin, 2014; Matos, Simões, Camacho, \& Reis, 2014). 


\section{CONSUMO DE TABACO, ÁLCOOL E DROGAS EM JOVENS ESTUDANTES}

Foi verificada associação entre o consumo de tabaco com a existência de pais fumadores e também com a presença de outras pessoas fumadoras. 0 consumo de tabaco por parte dos pais, irmãos e amigos, é também considerado fator potenciador do consumo de tabaco (American Academy of Pediatrics, 2001; Tavares, Béria \& Lima, 2004). As amizades constituem um enorme reforço ou determinante no ao consumo de substâncias, podendo constituir um reforço a presença especial dos chamados amigos íntimos (Prinstein, Boergers \& Spirito, 2001).

0 álcool constitui a substância psicoativa mais consumida e simultaneamente a droga de eleição entre crianças e adolescentes, o que é corroborado pela Organização Mundial de Saúde (WHO, 2001; WHO, 2004).

Foi verificada também associação entre o consumo de álcool e tabaco com o já ter iniciado relações sexuais. Os consumos aumentam potencialmente nos adolescentes que têm outros comportamentos de risco (American Academy of Pediatrics, 2001).

\section{CONCLUSÃO}

0 êxito das intervenções em saúde depende de um bom diagnóstico. Os resultados da presente investigação identificam o papel da família e dos amigos como determinantes nos consumos de tabaco, álcool e drogas em estudantes adolescentes. Foi verificada associação direta entre o consumo nos adolescentes e a existência de pais consumidores.

\section{REFERÊNCIA BIBLIOGRÁFICAS}

American Academy of Pediatrics (2001). Committee on substance abuse tobacco's toll: implications for the pediatrician. Pediatrics, 107(4):794-8.

DGS (2006). Consultas de Vigilância de Saúde Infantil e Juvenil: Actualização das curvas de crescimento. Circular Normativa №: 05/DSMIA.

DiClemente, R., Hansen, W., \& Ponton, L. (Eds.). (1996). Handbook of adolescent health risk behavior. New York: Plenum Press.

Galduroz JC, Noto AR, Nappo SA, Carlini EA (2004) Trends in drug use among students in Brazil: analysis of four surveys in 1987, 1989, 1993 and 1997. Braz J Med Biol Res.;37(4):523-31.

Hershner, S. D. \& Chervin, R. D. (2014). Causes and consequences of sleepiness among college students. Nature and Science of Sleep 2014:6 73-84.

Hingson R, Winter M. Epidemiology and consequences of drinking and driving. Alcohol Res Health. 2003;27(1):63-78.

Lerner, R., \& Thompson, L. (2002). Promoting healthy adolescent behavior and development: issues in the design and evaluation of effective youth programs. Journal of Pediatric Nursing, 17 (5), 338-344.

Mallow-Iroff, M. (2006). Cross-sex best friendship influences on early adolescents' cigarette and alcohol expectancies and use. Journal of Psychology, 140 (83), 209-227.

Matos, M. et al. (2003). A saúde dos adolescentes Portugueses: 4 anos depois. Fundação da Ciência e Tecnologia e Faculdade de Motricidade Humana: Lisboa.

Matos, M.G. (2008). A saúde do adolescente: 0 que se sabe e quais são os novos desafios. Análise Psicológica: 2 (XXVI). 251-263.

Matos, M.G. (Ed.) (2005). Comunicação, gestão de conflitos e saúde na escola. Lisboa: CDI, Faculdade de Motricidade Humana.

Matos, M.G.(cord.). (2008b). Consumo de substâncias: Estilo de vida? À procura de um estilo? Lisboa, Instituto da Droga e da Toxicodependência.

Meloni JN, Laranjeira R. Custo social e de saúde do consumo do álcool. Rev Bras Psiquiatr. 2004;26(Supl1):7-10. 
Prinstein, M. J., Boergers, J., \& Spirito, A. (2001). Adolescents and their friends' health-risk behavior: Factors that alter or add to peer influence. Journal of Pediatric Psychology, 26 (5), 287-298.

Reboussin BA, Song EY, Shrestha A, Lohman KK, Wolfson M. (2006). A latent class analysis of underage problem drinking: evidence from a community sample of 16-20 year olds. Drug Alcohol Depend;83(3):199-209.

Simões C. Adolescentes e comportamentos de saúde. Alicerces. 2010;III(3):223-41.)

Vieira, D.L.; Ribeiro, M.; Romano, M. \& Laranjeira, R.R. (2007). Álcool e adolescentes: estudo para implementar políticas municipais. Rev Saúde Pública: 41(3):396-403.

WHO 2001. Global status report: alcohol and young people. Geneva

WHO 2004. Global status report on alcohol 2004. Geneva:.20,21 
